\title{
Heat Transfer, Newton's Law of Cooling and the Law of Entropy Increase Simulated by the Real-Time Computer Experiment in Java
}

\author{
Adam Galant, Ryszard Kutner, and Andrzej Majerowski \\ Institute of Experimental Physics, Department of Physics, Warsaw University Hoża \\ 69, PL-00681 Warsaw, Poland
}

\begin{abstract}
Exploitation of a real-time computer experiment ${ }^{1}$ connected with advanced education of statistical mechanics and thermodynamics allows us to study a commonly occurring phenomenon of heat transfer through a diathermic wall; this wall separates the container into two parts consisting of an ideal gas at different temperatures. The system as a whole is isolated from the surroundigs by very massive border walls. We assume that the walls are rough which introduces the necessary spatial molecular chaos in the system. We study (i) the thermalization process where the temperatures of both parts of the container relax to the same, final one. We found (ii) that the temperature difference relaxes according to the empirical Newton's law of cooling obeyed by real systems. Finally, we confirmed (iii) the law of entropy increase and verified its validity for this small system. We used a simple hybrid molecular dynamics where the scattering of atoms has a random character but between the successive scattering steps the motion of atoms is deterministic.
\end{abstract}

\section{Introduction}

The second law of thermodynamics is still a subject of intensive study [12] by physical, mathematical and numerical means in the context of chaotic [23] and/or small systems, molecular motors, living organisms, theory of information [3, systems in extreme conditions and/or far from the stationary (or equilibrium) state called also nonlinear nonequilibrium and fluctuation-dissipative thermodynamics [4]. Another, quickly developing branch of statistical physics is the non-extensive thermodynamics which generalizes the second law of thermodynamics and the classical definition of entropy [5. The validity of the principles of thermodynamics is still verified the more so as there are several almost (but not fully) equivalent formulations of the second law of thermodynamics. The principal open question is whether the second law of thermodynamics is a basic law or a coarse-grain one which somehow follows from more fundamental ones? In other words, what is the relation between the macroscopic description of nature and its microscopic foundation? Hence, one is justified to pose the question

\footnotetext{
${ }^{1}$ This computer experiment is the Java applet located under the internet address http://tempac.fuw.edu.pl/erka/clausius/Clausius.html.
} 
whether complexity and irreversability are a new quality of matter or only an aspect of microscopic laws 67]?

Quite often, the law of entropy increase is presented by experiments related to expansion or mixing of (ideal or real) gases [89]. However in this work we considered, by a dynamical computer experiment, the more complex process of heat transfer which as yet is the most important one both for reversible and irreversible thermodynamics.

The obtained results are affected by fluctuations which, fortunately, for more than several dozen atoms are sufficiently small to allow observing the expected trends in the data. Our computer experiment gives students the opportunity to currently view (during the lecture) the microscopic realization of the heat transfer process being the most important one for the statistical mechanics and thermodynamics.

\section{Model and Hybrid Algorithm}

We define the model together with the hybrid algorithm which allows us to simulate and visualize in real time as the Java applet, the evolution of quantities relevant to nonequilibrium and equilibrium statistical mechanics and thermodynamics. We use the algorithm where the simplest version of the molecular dynamics is mixed with the simplest 'hit and miss' Monte Carlo recipe. This hybrid approach can be an example of the Wolfram idea 10 saying that already simple computer programs can model a complex behaviour of physical sytems, especially their evolution, which well describes the essential features of the empirical ones.

Preparation of the initial state. We consider the model of an ideal gas (i.e., a gas composed of classical, noninteracting, identical point particles) located in a container isolated from the surroundigs by very massive border walls. The container is devided into two parts (of equal volume) by a diathermic wall which makes possible heat transfer between them (c.f. Figs 1 and 2). This means that both parts of the container are in thermal contact 1112 and therefore particles can indirectly interact. For an ideal gas the assumption is justified that initially the kinetic energy of the particles is the same in each part of the container although the orientations of the velocity vector of the particles are random (other distributions of kinetic energy, for example the Maxwell one, are also possible but it does not seem necessary to use them for the ideal gas).

The gas contained in the left part consists of $N_{L}$ particles and has initially the temperature $T_{0}^{L}$; we have the same for the right part. In general $T_{0}^{L} \neq T_{0}^{R}$ and $N_{L} \neq N_{R}$ (all these quantities can be introduced at the beginning of the computer experiment). The initial temperatures are simply defined by using the kinetic energy of particles $\varepsilon_{0}^{j}, j=1,2, \ldots, N_{L}+N_{R}$,

$$
k_{B} T_{0}^{L, R}=\varepsilon_{0}^{j}=\frac{\left(p_{0}^{j, x}\right)^{2}+\left(p_{0}^{j, y}\right)^{2}}{2 \mu},
$$




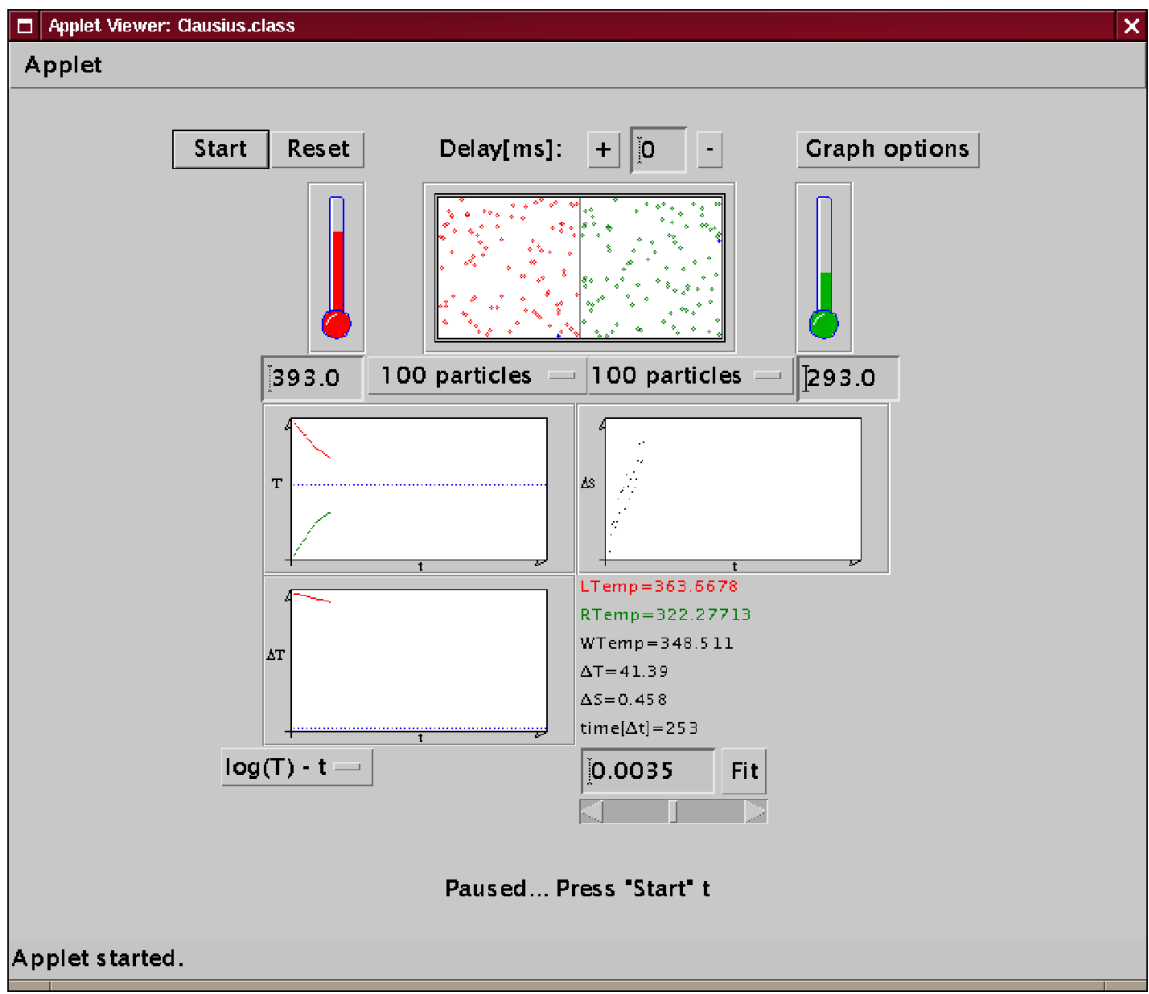

Fig. 1. The intermediate stage of heat transfer through the diathermic wall (the upper window). Results of the system evolution after 253 time steps are shown for temperatures of both parts of the container (the middle window), for the difference of both temperatures (the lowest window) and the change of entropy of the system (the right middle window); note that the lowest window has a semi-logarithmic scale while the two remaining ones have linear scales.

where $k_{B}$ is the Boltzmann constant, $\mu$ is the mass of any particle and $p_{0}^{j, x}, p_{0}^{j, y}$ are its (planar) momentum components. Both particle numbers $N_{L}$ and $N_{R}$ are placed directly below the respective parts of the container while temperatures are placed below the red and green thermometers, respectively, shown in Figs.1. and 2 additionaly these temperatures (as well as the temperature of the diathermic wall defined below) are given as the initial values in the dynamic table shown in the same figures (and described in Sec.3).

The initial temperature of the diathermic wall is defined as proportional to the average kinetic anergy of all particles

$$
k_{B} T_{0}^{w}=\frac{1}{N}\left(\sum_{j=1}^{N_{L}} \varepsilon_{0}^{j}+\sum_{j=N_{L}+1}^{N} \varepsilon_{0}^{j}\right)
$$




\section{Applet}

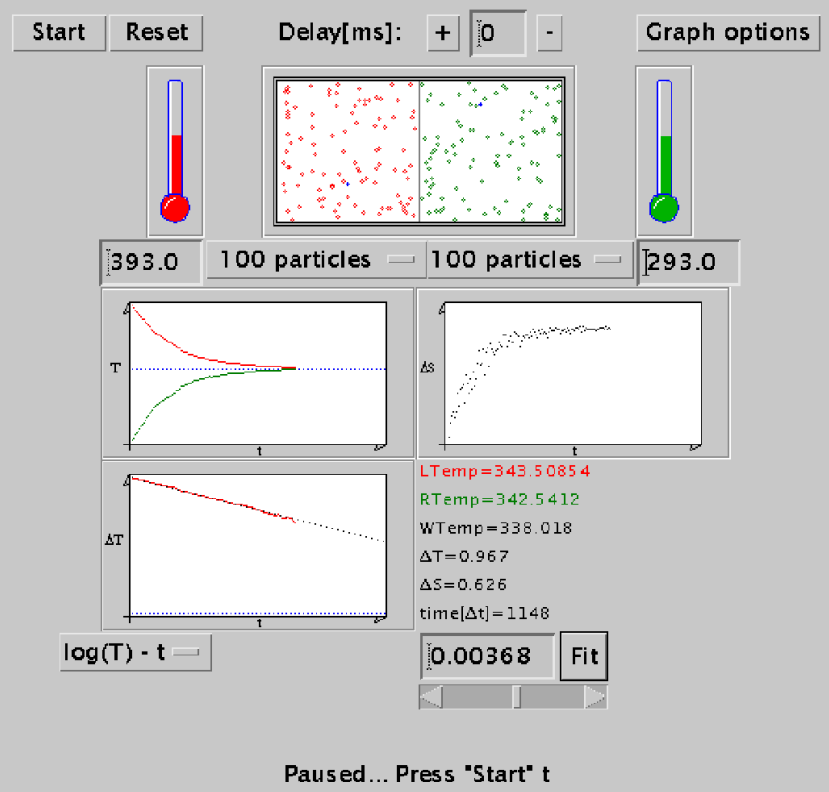

Applet started.

Fig. 2. Almost final stage of the heat transfer through the diathermic wall as results of the system evolution (after 1148 time steps) are shown until the temperature difference was not greater than $1 K$; note that the lowest window has a semi-logarithmic scale while the two remaining ones have linear scales.

$$
=k_{B}\left(\frac{N_{L}}{N} T_{0}^{L}+\frac{N_{R}}{N} T_{0}^{R}\right)
$$

where $N=N_{L}+N_{R}$ is the total number of particles.

We prove in Sec 3 that expression (2) also defines a temperature neutral diathermic wall, i.e., a wall whose temperature can only fluctuates but doesn't systematically change.

Indirect interaction between particles. To develop the stochastic dynamics of the system we devided our procedure into several stages. The first stage is a

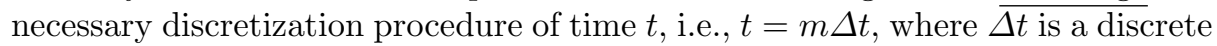
time-step and $m=1,2, \ldots$, is the number of the successive steps.

In the second stage we consider an inelastic scattering of particles by the diathermic wall; only in this way the particles can indirectly interact between themselves. We assume that within a given time interval $\Delta t$ exactly $n_{L}\left(\leq N_{L}\right)$ particles from the left part of the container and $n_{R}\left(\leq N_{R}\right)$ particles from the 
right one interact with the diathermic wall (both $n_{L}$ and $n_{R}$ are fluctuating $m$ dependent numbers). If we denote the internal energy of the diathermic wall in the $m^{t h}$ time-step by $\varepsilon_{m}^{w}$ and the corresponding kinetic energies of particles by

$$
\varepsilon_{m}^{j}=\frac{\left(p_{m}^{j, x}\right)^{2}+\left(p_{m}^{j, y}\right)^{2}}{2 \mu}, j=1,2, \ldots, n_{L}+n_{R},
$$

we can find the energies of each interacting object in the next $m^{t h}+1$ time-step by applying the local principle of equipartition energy. Namely,

$$
\varepsilon_{m+1}=\frac{1}{n_{L}+n_{R}+1}\left[\sum_{j=1}^{n_{L}} \varepsilon_{m}^{j}+\sum_{j=n_{L}+1}^{n_{R}} \varepsilon_{m}^{j}+\varepsilon_{m}^{w}\right]
$$

which is already the same for all scattered particles and the wall; as it is seen, the diathermic wall is treated here as an additional particle (which can only oscillate due to reaction forces but is not subject to the translatory motion, i.e. its momentum is absorbed by the massive border walls). It should be emphasized that relation (4) is local in time and results from the energy transfer (in the form of heat); this transfer doesn't necessaryly proceed from the warmer to colder part of the container. The extension of our assumption in order that the diathermic wall would consist of $N_{w}$ particles is straightforward (then $\varepsilon_{m}^{w}$ should be interpreted as the total energy of $N_{w}$ particles and the denominator in expression (4) should be changed by $n_{L}+n_{R}+N_{w}$ ).

The surface of the diathermic wall is rough which means that the component of the particle momentum directly before scattering parallel to the surface differs, in general, from the analogous component directly after the scattering, in distinction to the mirror type of scattering. Hence, the momentum components of the $j^{\text {th }}$ particle $\left(j=1,2, \ldots, n_{L}+n_{R}\right)$ are given by

$$
\begin{gathered}
p_{m+1}^{j, x}=-\cos \left(\phi_{j}\right) \sqrt{2 \mu \varepsilon_{m+1}}, \\
p_{m+1}^{j, y}=\sin \left(\phi_{j}\right) \sqrt{2 \mu \varepsilon_{m+1}},
\end{gathered}
$$

where the scattering angle $\phi_{j}$ is a random variable, index $x$ denotes here the direction perpendicular to the surface, while $y$ the parallel direction (as the diathermic wall is vertical here, c.f. Figs 1 and 2).

In the third stage the position $\left(x_{m+1}^{j}, y_{m+1}^{j}\right), j=1,2, \ldots, n_{L}+n_{R} ; m=$

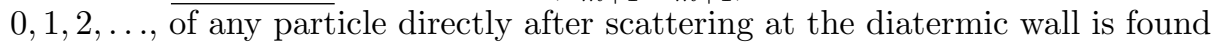
by the simple rule; the initial step of this rule is the division of the time interval $\Delta t=\Delta t_{1}+\Delta t_{2}$, where during time $\Delta t_{1}$ a particle reaches the wall while $\Delta t_{2}$ is the departure time from this wall. We can write,

$$
\Delta t_{1}=\frac{x^{w}-x_{m}^{j}}{p_{m}^{j, x} / \mu}
$$

independently of whether the particle is located in the left or right part of the container (here $x^{w}$ is the $x$-component of the position of the diathermic wall). 
Hence, the $x$-component of the particle position

$$
x_{m+1}^{j}=x^{w}+\Delta t_{2} \frac{p_{m+1}^{j, x}}{\mu}, j=1,2, \ldots, n_{L}+n_{R},
$$

where $\Delta t_{2}=\Delta t-\Delta t_{1}$, while the $y$-component is given by the relation

$$
y_{m+1}^{j}=y_{m}^{j}+\Delta t_{1} \frac{p_{m}^{j, y}}{\mu}+\Delta t_{2} \frac{p_{m+1}^{j, y}}{\mu} .
$$

As it is seen, from the knowledge of the state (i.e., the position and momentum) of the particle directly before the scattering at the diathermic wall we find its state directly after the scattering.

Scattering at the massive border wall. The interaction of any particle with this wall is elastic although the border wall is again rough. This means that relation (4) is violated while in expressions (5) we can assume $\varepsilon_{m+1}^{j}=\varepsilon_{m}^{j}, j=$ $1,2, \ldots, N_{L}+N_{R} ; m=0,1,2, \ldots$, i.e., directly before and after scattering of the particle at the massive border wall the energy of any scattered particle is the equal. Fortunately, expressions (6), (7) and (8) are, in principle, valid in this case but the following comment should be made. Namely, these relations regard the vertical border walls, where $x^{w}$ means here the $x$-component of their positions. For horizontal border walls relations analogous to (6), (7) and (8) are obeyed.

We should emphasize that though we deal with ideal gas two mechanisms of mixing have been introduced: (i) the space mixing of the particles as the scattering angle of any particle is random and (ii) the mixing based on the local principle of equipartition of energy which divides the energy between two parts of the container. Hence, we extended the possibility of application of the ideal gas model that can be used as the reference one, for example, for the Boltzmann gas, where binary collisions between particles are taken into account and the entropy is calculated analytically (in the approximated way) by using the Boltzmann kinetic equation and the $H$ function [13].

\section{Time-Dependent Thermodynamic Quantities}

In this work two types of thermodynamic quantities are calculated and visualized as a function of time: (i) temperatures of gases in both parts of the container and the difference between them and (ii) the change of entropy of the system. We assume that these quantities are well defined as far as they are subject to sufficiently small fluctuations so their main trend may be seen (which is particularly important for the change of the entropy; c.f. Figs 1 and 21.

The current temperature of the gas in each part of the container, $T_{m}^{L, R}$, in the $m^{t h}$ time-step $(m=0,1,2, \ldots$,$) is defined as proportional to the current$ average kinetic energy of particles in the given part, which is consistent with definitions (11) and (3)

$$
k_{B} T_{m}^{L}=\frac{1}{N_{L}} \sum_{j=1}^{N_{L}} \varepsilon_{m}^{j}
$$




$$
k_{B} T_{m}^{R}=\frac{1}{N_{R}} \sum_{j=N_{L}+1}^{N_{L}+N_{R}} \varepsilon_{m}^{j}
$$

Similarly, for the diathermic wall we define

$$
k_{B} T_{m}^{w}=\varepsilon_{m}^{w}
$$

However, the current temperature of the diathermic wall doesn't systematically change and can only fluctuate (which can be easily seen by looking for the corresponding data presented during the computer experiment). This can be understood if we remember that the total energy $E$ of the system is preserved during its evolution; i.e., for any time-step $m(=0,1,2, \ldots$,$) we can write$

$$
E=\sum_{j=1}^{N} \varepsilon_{m}^{j}+\varepsilon_{m}^{w}=\text { const }
$$

Particularly, for $m \rightarrow \infty$ we obtain (by using (9) and (10)

$$
N_{L} T_{0}^{L}+N_{R} T_{0}^{R}+T_{0}^{w}=N_{L} T_{\infty}^{L}+N_{R} T_{\infty}^{R}+T_{\infty}^{w}
$$

Note that extension of (12) to a more general description where the diathermic wall consists of $N_{w}(>1)$ particles (then temperatures $T_{l}^{w}, l=m, 0, \infty$, should by replaced by $N_{w} T_{l}^{w}$ ) doesn't change the final result (15) given below.

Thermodynamic equilibrium is characterized by equality of all (final) temperatures, i.e.,

$$
T_{\infty}^{L}=T_{\infty}^{R}=T_{\infty}^{w}=T_{\infty}
$$

Moreover, we assume for simplicity the condition of temperature neutrality of the diathermic wall which requires

$$
T_{m}^{w}=T_{\infty}, m=0,1,2, \ldots,
$$

and leads, by using (12), (13) and (14), to expression

$$
T_{0}^{w}=T_{\infty}=\frac{N_{L}}{N} T_{0}^{L}+\frac{N_{R}}{N} T_{0}^{R}
$$

which is consistent with definition (2).

When the simulation begin, the temperatures of the left and right parts (which, in general, are initially different) tend to the same value, subject only to small fluctuations (this is already obeyed for the number of particles greater than several dozen in each part of the container) while the temperature of the diathermic wall fluctuates, sometimes even strongly, since this wall is treated as an additional particle and not a very massive one.

As it is seen from Figs[1] and [2 the dynamic table enclosed there concerns: (1) temporal temperatures of both parts of the container $L T e m p \equiv T_{m}^{L}$ and 
$R T e m p \equiv T_{m}^{R}$, where $m=0,1,2, \ldots,(2)$ temporal temperature of the diathermic wall $W T e m p \equiv T_{m}^{w}$, (3) temporal temperature difference $\Delta T \equiv \Delta T_{m}=T_{m}^{L}-T_{m}^{R}$, (4) the entropy difference $\Delta S \equiv \Delta S_{m}=S_{m}-S_{0}$, and 5) time $\equiv m \Delta t$. Hence, we can observe that the fluctuations of temperatures of both parts of the container are relatively small opposite to the temperature of the diathermic wall (as the latter is considered as an additional particle and not a very massive wall).

It should be emphasized that the difference between temperatures, $\Delta T_{m}$, of both parts of the container obeys well known the empirical Newton law of cooling,

$$
\Delta T(t)=\Delta T(0) \exp (-t / \tau),
$$

where $\tau$ is the relaxation time depending on thermodynamic parameters of the system. This law is obeyd by real substances but in our numerical experiment we are able to imitate it by using ideal gas with an additional mechanism of spatial mixing of particles.

The law of entropy increase. By using the general definition of the change of entropy we can write

$$
d S_{m}=\frac{d E_{m}^{L}}{T_{m}^{L}}+\frac{d E_{m}^{w}}{T_{m}^{w}}+\frac{d E_{m}^{R}}{T_{m}^{R}},
$$

where $d S_{m}=S_{m+1}-S_{m}$ is the change of entropy within time interval $\Delta t$, while $d E_{m}^{j}=E_{m+1}^{j}-E_{m}^{j}(j=L, R, w)$ is the energy change (within the same time interval) of the left and right parts of the container, and the diathermic wall, respectively.

As it is seen in Figs 1 and 2 the entropy difference $\Delta S_{m}$ strongly fluctuates which is caused not only by the strong fluctuations of the temperature of the diathermic wall but also by the strong fluctuations of its energy change as well as energy changes of the ideal gas particles interacting with diathermic wall. Nevertheless, the general trend of entropy increase is still well seen even for our small system being far from equilibrium. This is an important result pointing to the extended range of validity of the law of entropy increase.

\section{Concluding Remarks}

In this paper we reconsidered the ideal gas model extending its possibility to simulate empirical phenomena and confirming its usefulness for educational purposes both in statistical mechanics and thermodynamics. This was possible thanks to that two mechanisms of mixing were exploited:

(i) Random scattering of particles at rough walls.

(ii) Local energy equipartition at the diathermic wall.

There are several conlusions having both educational and professional character.

(1) By using the real-time computer experiment it was possible to currently visualize both the 'macroscopic' heat transfer process and simultaneously its microscopic realization. 
(2) By using this dual visualization we pointed out how energy is transferred in a heating manner.

(3) Within this computer experiment we presented the trend of temperatures to equilize when subsystems come into the thermal contact.

(4) In this experiment we discovered that the temperature difference obeys the empirical Newton's law of cooling.

(5) In this experiment we observed both the entropy increase and the entropy fluctuations as well as the elementary entropy change due to entropy changes of both parts of the container and the diathermic wall.

We hope that the considered computer experiment will be an example that stimulates students to conduct their own experimentations for deeper understanding of physical phenomena.

\section{References}

1. Prigogine I., Stengers I.: Order Out of Chaos: Man's New Dialogue with Nature. Bantam Books, Toronto New York London Sydney (1984)

2. Zaslavsky G.M.: Chaotic dynamics and the origin of statitical laws. Physics Today 52 (1999) 39-45

3. Beck C., Schlögl F.: Thermodynamics of chaotic systems. Cambridge University Press, Cambridge (1995); Dorfman J.R.: An Introduction to Chaos in Nonequilibrium Statistical Mechanics. Cambridge University Press, Cambridge (1999); Schuster H.G.: Deterministic Chaos. An Introduction. Second Revised Edition. VCH Verlagsgesellschaft, Weinheim Basel Cambridge New York (1988)

4. Stratonovich R.L.: Nonlinear Nonequilibrium Thermodynamics I. Springer-Verlag, Berlin Heidelberg New York London Paris Tokyo Hong Kong Barcelona Budapest (1992)

5. Tsallis C.: Possible Generalization of Boltzmann-Gibbs Statistics. J. Stat. Phys. 52 (1988) 479-487

6. Lebowitz J.L.: Microscopic Reversability and Macroscopic Behavior: Physical Explanations and Mathematical Derivations. In: Brey, J.J., Marro, J., Rubi, J.M., San Miguel, M. In: 25 Years of Non-Equilibrium Statistical Mechanics. Lecture Notes in Physics, Vol. 445. Spriger-Verlag, Berlin Heidelberg New York (1995) 1-21

7. Lieb E.H., Yngvason J.: The Physics and Mathematics of the Second Law of Thermodynamics. Phys. Rep. 310 (1999) 1-96

8. Reif F.: Statistical Physics. Berkeley Physics Course Vol.5. McGraw-Hill Book Company, New York St. Louis San Francisco Toronto London Sydney (1967)

9. Atkins, P.A.: The Second Law. Scientific American Books. An Imprint of W.H. Freeman and Company, New York (1984)

10. Wolfram, S.: A New Kind of Science. Wolfram Media (2002)

11. Reif F.: Fundamental of Statistical and Thermal Physics. McGraw-Hill, New York San Francisco St. Louis Toronto London Sydney (1965)

12. Baierlein R.: Thermal Physics. Cambridge Univ. Press, Cambridge (1999)

13. Huang K.: Statistical Mechanics. J. Wiley and Sons, New York Chichster Brisbane Toronto Sigapore (1987) 